Pacific Journal of Mathematic 


\section{SYMMETRIC POSITIVE DEFINITE MULTILINEAR FUNCTIONALS WITH A GIVEN AUTOMORPHISM}

\section{Marvin Marcus and Stephen Pierce}

Let $V$ be an $n$-dimensional vector space over the real numbers $R$ and let $\varphi$ be a multilinear functional,

$$
\varphi: \stackrel{m}{x} V \longrightarrow R
$$

i.e., $\varphi\left(x_{1}, \cdots, x_{m}\right)$ is linear in each $x_{j}$ separately, $j=1, \cdots, m$. Let $H$ be a subgroup of the symmetric group $S_{m}$. Then $\varphi$ is said to be symmetric with respect to $H$ if

$$
\varphi\left(x_{\sigma(1)}, \cdots, x_{\sigma(m)}\right)=\varphi\left(x_{1}, \cdots, x_{m}\right)
$$

for all $\sigma \in H$ and all $x_{j} \in V, j=1, \cdots, m$. (In general, the range of $\varphi$ may be a subset of some vector space over $R$.) Let $T: V \rightarrow V$ be a linear transformation. Then $T$ is an automorphism with respect to $\varphi$ if

$$
\varphi\left(T x_{1}, \cdots, T x_{m}\right)=\varphi\left(x_{1}, \cdots, x_{m}\right)
$$

for all $x_{i} \in V, i=1, \cdots, m$. It is easy to verify that the set $\mathfrak{U}(H, T)$ of all $\varphi$ which are symmetric with respect to $H$ and which satisfy (3) constitutes a subspace of the space of all multilinear functionals symmetric with respect to $H$. We denote this latter set by $M_{m}(V, H, R)$.

We shall say that $\varphi$ is positive definite if

$$
\varphi(x, \cdots, x)>0
$$

for all nonzero $x$ in $V$, and we shall denote the set of all positive definite $\varphi$ in $\mathfrak{A}(H, T)$ by $P(H, T)$. It can be readily verified that $P(H, T)$ is a convex cone in $\mathfrak{A}(H, T)$.

Our main results follow.

THEOREM 1. If $P(H, T)$ is nonempty then and

(a) $m$ is even

(b) every eigenvalue of $T$ has modulus 1 . If, in addition, $T$ has only real eigenvalues then

(c) every elementary divisor of $T$ is linear. Conversely if (a), (b) and (c) hold then $P(H, T)$ is nonempty. Moreover, if $P(H, T)$ is nonempty then $\mathfrak{A}(H, T)$ is the linear closure of $P(H, T)$.

In Theorem 2 we shall investigate the dimension of $\mathfrak{A}(H, T)$ in the event $P(H, T)$ is not empty. To do this we must introduce some combinatorial notation. Let $\Gamma_{m, n}$ denote the set of all sequences 
$\omega=\left(\omega_{1}, \cdots, \omega_{m}\right)$ of length $m, 1 \leqq \omega_{i} \leqq n, i=1, \cdots, m$. Introduce an equivalence relation $\sim$ in $\Gamma_{m, n}$ as follows: $\alpha \sim \beta$ if there exists a $\sigma \in H$ such that

$$
\alpha^{\sigma}=\beta
$$

where $\alpha^{\sigma}=\left(\alpha_{\sigma(1)}, \cdots, \alpha_{\sigma(m)}\right)$. Let $\Delta$ be a system of distinct representatives for $\sim$, i.e., $\Delta$ is a set of sequences, one from each equivalence class with respect to $\sim$. We specify $A$ uniquely by choosing each element $\alpha \in \Delta$ to be lowest in lexicographic order in the equivalence class in which $\alpha$ occurs.

THeOREM 2. If $P(H, T)$ is nonempty and $T$ has real eigenvalues $\gamma_{1}, \cdots, \gamma_{n}$ then $\gamma_{i}= \pm 1, i=1, \cdots, n$. Suppose

$$
\gamma_{i_{1}}=\cdots=\gamma_{i_{p}}=1, \quad \gamma_{j}=-1, \quad j \neq i_{1}, \cdots, i_{p} .
$$

Let $\mu_{p}$ be the number of sequences $\omega$ in $\Delta$ such that the total number of occurrences of $i_{1}, \cdots, i_{p}$ in $\omega$ is even. Then

$$
\operatorname{dim} \mathfrak{A}(H, T)=\mu_{p} .
$$

Corollary. If $H=S_{m}$ in Theorem 2 and $T$ has $p$ eigenvalues 1 and $n-p$ eigenvalues -1 then

$$
\operatorname{dim} \mathfrak{Y}(H, T)=\sum_{k=0}^{m / 2}\left(\begin{array}{c}
p-1+2 k \\
p-1
\end{array}\right)\left(\begin{array}{c}
n-p-1+m-2 k \\
n-p-1
\end{array}\right) .
$$

In case $m=2, H=S_{2}, \mathfrak{A}(H, T)$ is the totality of symmetric bilinear functionals $\varphi$ for which

$$
\varphi\left(T x_{1}, T x_{2}\right)=\varphi\left(x_{1}, x_{2}\right), \quad x_{1}, x_{2} \in V,
$$

and $P(H, T)$ is just the cone of positive definite $\rho$ in $\mathfrak{U}(H, T)$ i.e.,

$$
\varphi(x, x) \geqq 0
$$

with equality only if $x=0$. In this case we need not assume that $T$ has real eigenvalues in order to analyze $\mathfrak{A}(H, T)$. We can easily prove the following result by our methods, most parts of which are known (see e.g. [1], Chapter 7).

Theorem 3. Assume that $m=2$ and $H=S_{2}$. Then $P(H, T)$ is nonempty if and only if

(a) $T$ has linear elementary divisors over the complex field,

(b) every eigenvalue of $T$ has modulus 1. Suppose that $T$ has distinct complex eigenvalues

$$
\gamma_{k}=a_{k}+i b_{k} \quad\left(\text { and } \bar{\gamma}_{k}=a_{k}-i b_{k}\right)
$$


of multiplicity $e_{k}, k=1, \cdots, p$ and real eigenvalues

$$
\gamma_{k}=r_{k}, \quad k=\sum_{j=1}^{p} 2 e_{j}+1, \cdots, n .
$$

If $P(H, T)$ is nonempty then the elementary divisors of $T$ over the real field are

$$
\begin{aligned}
\lambda^{2}-2 \lambda a_{k}+1, & e_{k} \text { times, } \quad k=1, \cdots, p, \\
\lambda-1, & q \text { times, } \\
\lambda+1, & l \text { times, }
\end{aligned}
$$

where

$$
\sum_{j=1}^{p} 2 e_{j}+q+l=n .
$$

Moreover, $\mathfrak{Y}(H, T)$ is the linear closure of $P(H, T)$,

$$
\operatorname{dim} \mathfrak{A}(H, T)=\frac{q(q+1)}{2}+\frac{l(l+1)}{2}+\sum_{j=1}^{p} e_{j}^{2},
$$

and there exists a basis $E$ of $V$ such that $\mathfrak{A}(H, T)$ consists of the set of all $\phi$ whose matrix representations with respect to $E,[\varphi]_{E}^{E}$, have the following form:

$$
[\varphi]_{E}^{E}=\sum_{k=1}^{p} \cdot\left(X_{k} \otimes I_{2}+Y_{k} \otimes F\right)+H_{q}+H_{l} .
$$

In (6), the dot indicates direct sum, $\otimes$ denotes the Kronecker product, $F=\left[\begin{array}{cc}0 & 1 \\ -1 & 0\end{array}\right], X_{k}$ is $e_{k}$-square symmetric, $Y_{k}$ is $e_{k}$-square skew-symmetric, $H_{q}$ and $H_{l}$ are $q$-square and $l$-square symmetric respectively.

2. Proofs. Let $V^{m}(H)$ denote the symmetry class of tensors associated with $H$ [2]. That is, there exists a fixed multilinear function $\tau: \mathbf{X}_{1}^{m} V \rightarrow V^{m}(H)$ symmetric with respect to $H$, for which

(i) the linear closure of $\tau\left(\mathbf{X}_{1}^{m} V\right)$ is $V^{m}(H)$

(ii) the pair $\left(V^{m}(H), \tau\right)$ is universal: given any space $U$ and any multilinear function $\varphi: \mathrm{X}_{1}^{n} V \rightarrow U$ symmetric with respect to $H$, there exists a (unique) linear $h_{\varphi}: V^{m}(H) \rightarrow U$ satisfying

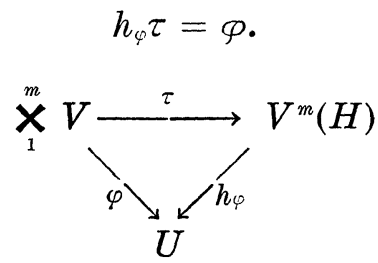


We shall denote $\tau\left(x_{1}, \cdots, x_{m}\right)$ by $x_{1} * \cdots * x_{m}$, and $x_{1} * \cdots * x_{m}$ is called a decomposable tensor or a symmetric product of $x_{1}, \cdots, x_{m}$. If we take $\varphi\left(x_{1}, \cdots, x_{m}\right)$ to be $T x_{1} * \cdots * T x_{m}$ in (7) then $h_{\varphi}$ is denoted by $K(T)$ and is called the induced transformation on $V^{m}(H)$.

Before we embark on the proof of Theorem 1 we can define $\mathfrak{A}(H, T)$ in terms of $V^{m}(H)$. First observe that the mapping $\theta$ from the space of multilinear functionals $\varphi$ symmetric with respect to $H$ to the dual space of $V^{m}(H)$,

$$
\theta: M_{m}(V, H, R) \longrightarrow\left(V^{m}(H)\right)^{*},
$$

defined by

$$
\theta(\varphi)=h_{\varphi},
$$

is one-to-one linear, and onto. That is, the correspondence $\varphi \leftrightarrow h_{\varphi}$ is linear bijective. Now, the subspace $\mathfrak{H}(H, T)$ of $M_{m}(V, H, R)$ is defined by

$$
\varphi\left(T x_{1}, \cdots, T x_{m}\right)=\varphi\left(x_{1}, \cdots, x_{m}\right)
$$

or in view of (7) by

$$
h_{\varphi}\left(T x_{1} * \cdots * T x_{m}\right)=h_{\varphi}\left(x_{1} * \cdots * x_{m}\right),
$$

for all $x_{i} \in V, i=1, \cdots, m$. In other words, since the decomposable tensors span $V^{m}(H)$ (see (i) above), $\varphi \in \mathfrak{A}(H, T)$ if and only if $\theta(\varphi)=h_{\varphi}$ satisfies

$$
h_{\varphi} K(T)=h_{\varphi},
$$

or

$$
h_{\varphi}(K(T)-I)=0
$$

where $I$ is the identity mapping on $V^{m}(H)$. We have proved the following.

LeMma 1. $\mathfrak{A}(H, T)$ is nonempty if and only if $K(T)-I$ is singular. Also,

$$
\operatorname{dim} \mathfrak{A}(H, T)=\eta(K(T)-I)
$$

where $\eta$ is the nullity of the indicated transformation.

Lemma 2. If $P(H, T)$ is nonempty then $m$ is even and every eigenvalue of $T$ has modulus 1 . Moreover, corresponding to real eigenvalues, $T$ has only linear elementary divisors.

Proof. If $\varphi \in P(H, T)$ and $x \neq 0$ then 


$$
\varphi(-x, \cdots,-x)=(-1)^{m} \varphi(x, \cdots, x)
$$

and hence $(-1)^{m}>0$ and $m$ is even. Suppose that $\gamma$ is a real eigenvalue of $T$ with corresponding eigenvector $x$. Then

$$
\begin{aligned}
\varphi(T x, \cdots, T x) & =\varphi(\gamma x, \cdots, \gamma x) \\
& =\gamma^{m} \varphi(x, \cdots, x) .
\end{aligned}
$$

Since $\varphi \in P(H, T), \varphi(T x, \cdots, T x)=\varphi(x, \cdots, x)>0$ and hence $\gamma^{m}=1$ and $\gamma= \pm 1$. If $\gamma$ were involved in an elementary divisor of degree greater than 1 then there would exist linearly independent vectors $u_{1}$ and $u_{2}$ such that $T u_{1}=\gamma u_{1}, T u_{2}=\gamma u_{2}+u_{1}$ and hence

$$
\varphi\left(T u_{1}, \cdots, T u_{1}, T u_{2}\right)=\varphi\left(\gamma u_{1}, \cdots, \gamma u_{1}, \gamma u_{2}+u_{1}\right) .
$$

Now

$$
\begin{aligned}
\varphi\left(u_{1}, \cdots, u_{1}, u_{2}\right) & =\gamma^{m} \varphi\left(u_{1}, \cdots, u_{1}, u_{2}\right) \\
& =\varphi\left(\gamma u_{1}, \cdots, \gamma u_{1}, \gamma u_{2}\right)
\end{aligned}
$$

so that

$$
\begin{aligned}
0 & =\varphi\left(\gamma u_{1}, \cdots, \gamma u_{1}, \gamma u_{2}+u_{1}\right)-\varphi\left(\gamma u_{1}, \cdots, \gamma u_{1}, \gamma u_{2}\right) \\
& =\varphi\left(\gamma u_{1}, \cdots, \gamma u_{1}, u_{1}\right) \\
& =\gamma^{m-1} \varphi\left(u_{1}, \cdots, u_{1}\right),
\end{aligned}
$$

a contradiction.

We now show that any complex eigenvalue of $T$ has modulus 1 . Since $\gamma=a+i b$ is now assumed not to be real there exists a pair of linearly independent vectors $v_{1}$ and $v_{2}$ in $V$ such that

$$
\begin{aligned}
& T v_{1}=a v_{1}-b v_{2} \\
& T v_{2}=b v_{1}+a v_{2} .
\end{aligned}
$$

Let $\vec{V}$ be the extension of $V$ to an $n$-dimensional space over the complex field. Now $\varphi \in \mathfrak{A}(H, T)$ means that

$$
\varphi\left(T x_{1}, \cdots, T x_{m}\right)-\varphi\left(x_{1}, \cdots, x_{m}\right)=0
$$

is an identity in $x_{1}, \cdots, x_{m}$. If we express the vectors in $\bar{V}$ in terms of a basis in $V$ (using in general complex rather than real coefficients) the identity (11) continues to hold since it is a homogeneous polynomial of degree $m$ in the components of $x_{1}, \cdots, x_{m}$, vanishing for all real values of these components. Of course it is not true that

$$
\varphi(x, \cdots, x)>0
$$

continues to hold for nonzero $x \in \bar{V}$. Now define 


$$
\begin{aligned}
& e_{1}=v_{1}+i v_{2} \in \bar{V} \\
& e_{2}=v_{1}-i v_{2} \in \bar{V}
\end{aligned}
$$

and observe that $e_{1}$ and $e_{2}$ are linearly independent in $\bar{V}$ and satisfy

$$
\begin{aligned}
& T e_{1}=\gamma e_{1} \\
& T e_{2}=\bar{\gamma} e_{2} .
\end{aligned}
$$

Let $\omega=\left(\omega_{1}, \cdots, \omega_{m}\right)$ be a sequence for which each $\omega_{i}$ is either 1 or $2, i=1, \cdots, m$ :

$$
\varphi\left(T e_{\omega_{1}}, \cdots, T e_{\omega_{m}}\right)=\gamma^{k} \bar{\gamma}^{m-k} \varphi\left(e_{\omega_{1}}, \cdots, e_{\omega_{m}}\right),
$$

where $k$ of the $\omega_{i}$ are 1 and $m-k$ are 2 . But by the above remarks

$$
\varphi\left(T e_{\omega_{1}}, \cdots, T e_{\omega_{m}}\right)=\varphi\left(e_{\omega_{1}}, \cdots, e_{\omega_{m}}\right)
$$

and taking absolute values we have

$$
\left(|\gamma|^{m}-1\right)\left|\varphi\left(e_{\omega_{1}}, \cdots, e_{\omega_{m}}\right)\right|=0 .
$$

Thus if $|\gamma| \neq 1$ it follows that

$$
\varphi\left(e_{\omega_{1}}, \cdots, e_{\omega_{m}}\right)=0
$$

for all $\omega$ for which $\omega_{i}$ is 1 or 2 for $i=1, \cdots, m$. From (12) we have $v_{1}=\left(e_{1}+e_{2}\right) / 2$ and hence using (13) we see that

$$
\begin{aligned}
\varphi\left(v_{1}, \cdots, v_{1}\right) & =\varphi\left(\frac{e_{1}+e_{2}}{2}, \cdots, \frac{e_{1}+e_{2}}{2}\right) \\
& =0 .
\end{aligned}
$$

However $v_{1} \in V$ and $\varphi \in P(H, T)$ and therefore (14) is a contradiction. Thus $|\gamma|=1$ and the proof of Lemma 2 is complete.

Lemma 3. If $m$ is even, and $T$ has real eigenvalues $r_{1}, \cdots, r_{n}$, and every elementary divisor of $T$ is linear then $P(H, T)$ is nonempty.

Proof. Since $T$ has linear elementary divisors there exists a basis for $V$ of eigenvectors $e_{1}, \cdots, e_{n}$. Let $g_{1}, \cdots, g_{n}$ be a dual basis in $V^{*}$. Let $g_{t}^{m}$ denote the multilinear functional whose value for any $x_{1}, \cdots, x_{m}$ in $V$ is

$$
\prod_{j=1}^{m} g_{t}\left(x_{j}\right) \text {. }
$$


Clearly $g_{t}^{m} \in M_{m}(V, H, R)$. Set

$$
\varphi=\sum_{t=1}^{n} g_{t}^{m}
$$

Then if $x_{j}=\sum_{k=1}^{n} \xi_{j k} e_{k}, j=1, \cdots, m$, and $T e_{k}=r_{k} e_{k}, k=1, \cdots, n$,

$$
\begin{aligned}
\varphi\left(T x_{1}, \cdots, T x_{m}\right) & =\sum_{t=1}^{n} \prod_{j=1}^{m} g_{t}\left(T x_{j}\right) \\
& =\sum_{t=1}^{n} \prod_{j=1}^{m} g_{t}\left(\sum_{k=1}^{n} \xi_{j k} T e_{k}\right) \\
& =\sum_{t=1}^{n} \prod_{j=1}^{m} \xi_{j t} r_{t} \\
& =\sum_{t=1}^{n} r_{t}^{m} \prod_{j=1}^{m} \xi_{j t} \\
& =\sum_{t=1}^{n} \prod_{j=1}^{m} \xi_{j t} \\
& =\sum_{t=1}^{n} \prod_{j=1}^{m} g_{t}\left(x_{j}\right) \\
& =\varphi\left(x_{1}, \cdots, x_{m}\right)
\end{aligned}
$$

Hence $\varphi \in \mathfrak{X}(H, T)$. Moreover, if $x=\sum_{t=1}^{n} c_{t} e_{t}$ then

$$
\begin{aligned}
\varphi(x, \cdots, x) & =\sum_{t=1}^{n} g_{t}(x)^{m} \\
& =\sum_{t=1}^{n} c_{t}^{m} .
\end{aligned}
$$

But $m$ is even and hence $\varphi \in P(H, T)$. To complete the proof of Theorem 1 we note that if $\varphi \in P(H, T)$ and if $e_{1}, \cdots, e_{n}$ is any basis of $V$ then $\varphi(x, x, \cdots, x)$ is a homogeneous polynomial of degree $m$ in $c_{1}, \cdots, c_{n}$. Hence, on the compact hypersphere $S$ defined by $\sum_{t=1}^{n} c_{t}^{2}=1$ in $V, \varphi$ must assume a positive minimum value $m_{\varphi}$. By a similar argument for any $\psi \in \mathfrak{A}(H, T),|\psi|$ must assume a maximum $M_{\psi}$ for $\sum_{t=1}^{n} c_{t}^{2}=1$. Now let $\psi$ be an arbitrary element of $\mathfrak{A}(H, T)$ and choose a positive constant $a$ such that $a>M_{\psi} / m_{\varphi}$. If $0 \neq x \in V$ and $\|x\|^{2}=$ $\sum_{t=1}^{n} c_{t}^{2}$ then $(x /\|x\|) \in S$ and

$$
\begin{aligned}
a \varphi(x, \cdots, x)-\psi(x, \cdots, x)= & a\|x\|^{m} \varphi\left(\frac{x}{\|x\|}, \cdots, \frac{x}{\|x\|}\right) \\
& -\|x\|^{m} \psi\left(\frac{x}{\|x\|}, \cdots, \frac{x}{" x \|}\right) \\
\geqq & \|x\|^{m}\left(a m_{\varphi}-M_{\psi}\right) \\
> & 0 .
\end{aligned}
$$

In other words, 


$$
a \varphi-\psi \in P(H, T)
$$

so that $\psi$ is a linear combination of elements in $P(H, T)$.

To proceed to the proof of Theorem 2 we use Theorem 1 to conclude immediately that since $T$ has real eigenvalues the elementary divisors are all linear and thus there exists a basis of eigenvectors of $T$ :

$$
T e_{k}=\gamma_{k} e_{k}, \quad k=1, \cdots, n \text {. }
$$

It is not difficult to show [2] that the decomposable tensors

$$
e_{\omega}^{*}=e_{\omega_{1}} * \cdots * e_{\omega_{m}}, \quad \omega \in \Delta,
$$

constitute a basis for $V^{m}(H)$.

We compute that

$$
\begin{aligned}
K(T) e_{\omega}^{*} & =T e_{\omega_{1}} * \cdots * T e_{\omega_{m}} \\
& =\gamma_{\omega_{1}} e_{\omega_{1}} * \cdots * \gamma_{\omega_{m}} e_{\omega_{m}} \\
& =\prod_{t=1}^{n} \gamma_{t}^{m} t^{(\omega)} e_{\omega}^{*}
\end{aligned}
$$

where $m_{t}(\omega)$ denotes the multiplicity of occurrence of $t$ in $\omega, t=$ $1, \cdots, n$. The formula (15) shows that $(K(T)-I) e_{\omega}^{*}$ is 0 or a nonzero multiple of $e_{\omega}^{*}$ according as

$$
\prod_{t=1}^{n} \gamma_{t}^{m_{t}}{ }^{(\omega)}
$$

is 1 or -1 . Now we can assume without loss of generality that the eigenvalues $\gamma_{1}, \cdots, \gamma_{n}$ are so organized that $\gamma_{1}=\cdots=\gamma_{p}=1, \gamma_{p+1}=$ $\cdots=\gamma_{n}=-1$. (This is of course merely a notational convenience.) Then

$$
\begin{aligned}
\prod_{t=1}^{n} \gamma_{t}^{m_{t}} t^{(\omega)} & =\prod_{t=p+1}^{n}(-1)^{m_{t}(\omega)} \\
& =(-1)^{m-\sum_{t=1}^{p} m_{t}(\omega)} \\
& =(-1) \sum_{t=1}^{p} m_{t}(\omega)
\end{aligned}
$$

Thus $\prod_{t=1}^{n} \gamma_{t}^{m} t^{(\omega)}=1$ if and only if $\sum_{t=1}^{p} m_{t}(\omega)$ is even. This last statement just means that $1, \cdots, p$ (i.e., $i_{1}, \cdots, i_{p}$ ) occur altogether an even number of times in $\omega$.

The proof of the corollary is completed by first noting that if $H=S_{m}$ then the set $\Delta$ is the totality of nondecreasing sequences of length $m$ chosen from $1, \cdots, n$. Thus by Theorem 2 if $P(H, T)$ is 
nonempty and $T$ has real eigenvalues $\gamma_{1}, \cdots, \gamma_{n}$ then these eigenvalues are \pm 1 and we lose no generality in assuming that $\gamma_{1}=\cdots=\gamma_{p}=1$, $\gamma_{p+1}=\cdots=\gamma_{n}=-1$. We want to count the total number of $\omega$ in $\Delta$ for which

$$
\sum_{t=1}^{p} m_{t}(\omega) \equiv 0 \quad(\bmod 2)
$$

Now, a sequence satisfying (16) may be constructed as follows. Suppose that $k$ is a fixed integer, $0 \leqq 2 k \leqq m$, and we count the number of sequences in $\Delta$ in which $\sum_{t=1}^{p} m_{t}(\omega)=2 k$. The total number of nondecreasing sequences of length $2 k$ using the integers $1, \cdots, p$ is

$$
\left(\begin{array}{c}
p+2 k-1 \\
2 k
\end{array}\right)=\left(\begin{array}{c}
p-1+2 k \\
p-1
\end{array}\right)
$$

and any one of these can be completed to a nondecreasing sequence of length $m$ by adjoining a nondecreasing sequence of length $m-2 k$ using the integers $p+1, \cdots, n$. There are a total of

$$
\left(\begin{array}{c}
n-p+m-2 k-1 \\
m-2 k
\end{array}\right)=\left(\begin{array}{c}
n-p-1+m-2 k \\
n-p-1
\end{array}\right)
$$

ways of doing this. This completes the proof of the corollary.

To proceed to the proof of Theorem 3 we remark that Theorem 1 cannot be directly applied because we are not assuming that the eigenvalues of $T$ are real; in general this is not the case. However the statement (b) does follow from Theorem 1 . If $E$ is any basis of $V, A$ is the matrix representation of $T$, and $C=[\varphi]_{E}^{E}$, then to say that $\varphi \in \mathfrak{A}(H, T)$ is equivalent to the assertion that

$$
A^{T} C A=C \text {. }
$$

If $\varphi \in P(H, T)$ then $C$ is a positive definite symmetric matrix and can therefore be written $C=K^{2}$, where $K$ is also positive definite symmetric. Then (17) is immediately equivalent to the statement that $K A K^{-1}$ is a real orthogonal matrix and (a) is evident. Conversely if (a) and (b) obtain then there exists a real nonsingular matrix $S$ such that $S^{-1} A S$ is a direct sum of a diagonal matrix with \pm 1 along the main diagonal together with certain 2-square matrices of the form

$$
\left[\begin{array}{cc}
a_{k} & b_{k} \\
-b_{k} & a_{k}
\end{array}\right]
$$

Since $\left|\gamma_{k}\right|=1, k=1, \cdots, n$, the matrix (18) is orthogonal and hence $S^{-1} A S=U$ where $U$ is an $n$-square real orthogonal matrix. If we set 
$\left(S^{T}\right)^{-1} S^{-1}=C$ then $C$ is a positive definite symmetric matrix and we compute that

$$
\begin{aligned}
A^{T} C A & =\left(S^{-1}\right)^{7} U^{T} S^{T}\left(S^{T}\right)^{-1} S^{-1} S U S^{-1} \\
& =\left(S^{-1}\right)^{T} S^{-1} \\
& =C .
\end{aligned}
$$

Thus if $[\varphi]_{L}^{E}=C$ then $\varphi \in P(H, T)$. The dimension of $\mathfrak{A}(H, T)$ can equally well be computed as in the general case by finding $\eta(K(T)-I)$ where $K(T)$ is the induced mapping on the complex space of 2-symmetric tensors over $\bar{V}$, i.e., $\bar{V}^{2}\left(S_{2}\right)$. The mapping $K(T)$ is just the 2 nd Kronecker power of $T$ restricted to the second symmetric space. This mapping is customarily denoted by $P_{2}(T)$ [5]. Since $T$ has a basis of eigenvectors $v_{1}, \cdots, v_{n}$, so does $P_{2}(T)$ and, for $1 \leqq i \leqq j \leqq n$,

$$
P_{2}(T) v_{i} * v_{j}=\gamma_{i} \gamma_{j} v_{i} * v_{j} \text {. }
$$

Thus $\operatorname{dim} \mathfrak{U}(H, T)$ is precisely the number of pairs of integers $(i, j)$, $1 \leqq i \leqq j \leqq n$, for which

$$
\gamma_{i} \gamma_{j}=1
$$

But $T$ has the distinct eigenvalues $a_{k}+i b_{k}$ of multiplicity $e_{k}, k=1, \cdots$, $p$. This yields a total of

$$
\sum_{t=1}^{p} e_{t}^{2}
$$

pairs $(i, j)$ for which $(19)$ is satisfied. Also, $T$ has 1 as an eigenvalue $q$ times and -1 as an eigenvalue $l$ times and this yields an additional

$$
\frac{q(q+1)}{2}+\frac{l(l+1)}{2}
$$

pairs $(i, j)$ for which (19) is satisfied. This proves that

$$
\operatorname{dim} \mathfrak{H}(H, T)=\frac{q(q+1)}{2}+\frac{l(l+1)}{2}+\sum_{j=1}^{p} e_{j}^{2} .
$$

We now turn to the derivation of (6). First, we assert that since $T$ has linear elementary divisors over the complex numbers [4] there exists a basis $E$ of $V$ such that the matrix representation of $T$ has the following form:

$$
A=\sum_{k=1}^{p} \cdot I_{e_{k}} \otimes\left[\begin{array}{cc}
a_{k} & b_{k} \\
-b_{k} & a_{k}
\end{array}\right]+I_{q}+-I_{l}
$$


where $I_{s}$ is the $s$-square identity matrix. We set $C=[\varphi]_{E}^{E}$ and partition $C$ conformally with (20):

$$
C=\left[\begin{array}{ccc|cc}
C_{11} & \cdots & C_{1 d} & & \\
\vdots & & \vdots & & \\
C_{d 1} & \cdots & C_{d d} & & \\
\hline & & C_{q} & C_{r} \\
& Z^{T} & & \\
& & C_{r}^{T} & C_{l}
\end{array}\right],
$$

$C_{i j}$ is 2-square, $i, j=1, \cdots, d=\sum_{j=1}^{p} e_{j}, C_{q}$ is $q$-square symmetric and $C_{l}$ is $l$-square symmetric. Set $L=\sum_{k=1}^{p \cdot} I_{e_{k}} \otimes\left(a_{k} I_{2}+b_{k} F\right)$ and observe that for (17) to be satisfied $Z$ must satisfy

$$
L^{T} Z\left(I_{q}+-I_{l}\right)=Z \text {. }
$$

Now, $L^{T} \otimes\left(I_{q}+-I_{l}\right)$ has eigenvalues $\pm\left(a_{k} \pm i b_{k}\right)[3$, p. 9] and none of these is equal to 1 . Hence (21) has only the zero matrix as a solution. Similarly we see that $C_{r}=0$. Next, consider a typical $C_{i j}, j>i$, call it $K$. Then $K$ must satisfy an equation of the form

$$
\left(a_{s} I_{2}-b_{s} F\right) K\left(a_{r} I_{2}+b_{r} F\right)=K \text {. }
$$

The matrix

$$
\left(a_{s} I_{2}-b_{s} F\right) \otimes\left(a_{r} I_{2}+b_{r} F\right)
$$

has eigenvalues

$$
\left(a_{s} \pm i b_{s}\right)\left(a_{r} \pm i b_{r}\right) \text {. }
$$

If $r \neq s$, (23) cannot be 1 and in this case $K=0$. If $r=s$ then precisely two of the four complex numbers (23) are 1 . Thus the nullity of the matrix

$$
\left(a_{s} I_{2}-b_{s} F\right) \otimes\left(a_{s} I_{2}+b_{s} F\right)-I_{4}
$$

is 2. But $K=I_{2}$ and $K=F$ are two linearly independent solutions to (22) for $r=s$. Also note that since $C$ is symmetric $C_{i i}$ must be a multiple of $I_{2}$. It follows that the submatrix

$$
\left[\begin{array}{ccc}
C_{11} & \cdots & C_{1 d} \\
\vdots & & \vdots \\
C_{d 1} & \cdots & C_{d d}
\end{array}\right]
$$

is itself a direct sum of $2 e_{k}$-square matrices of the form 


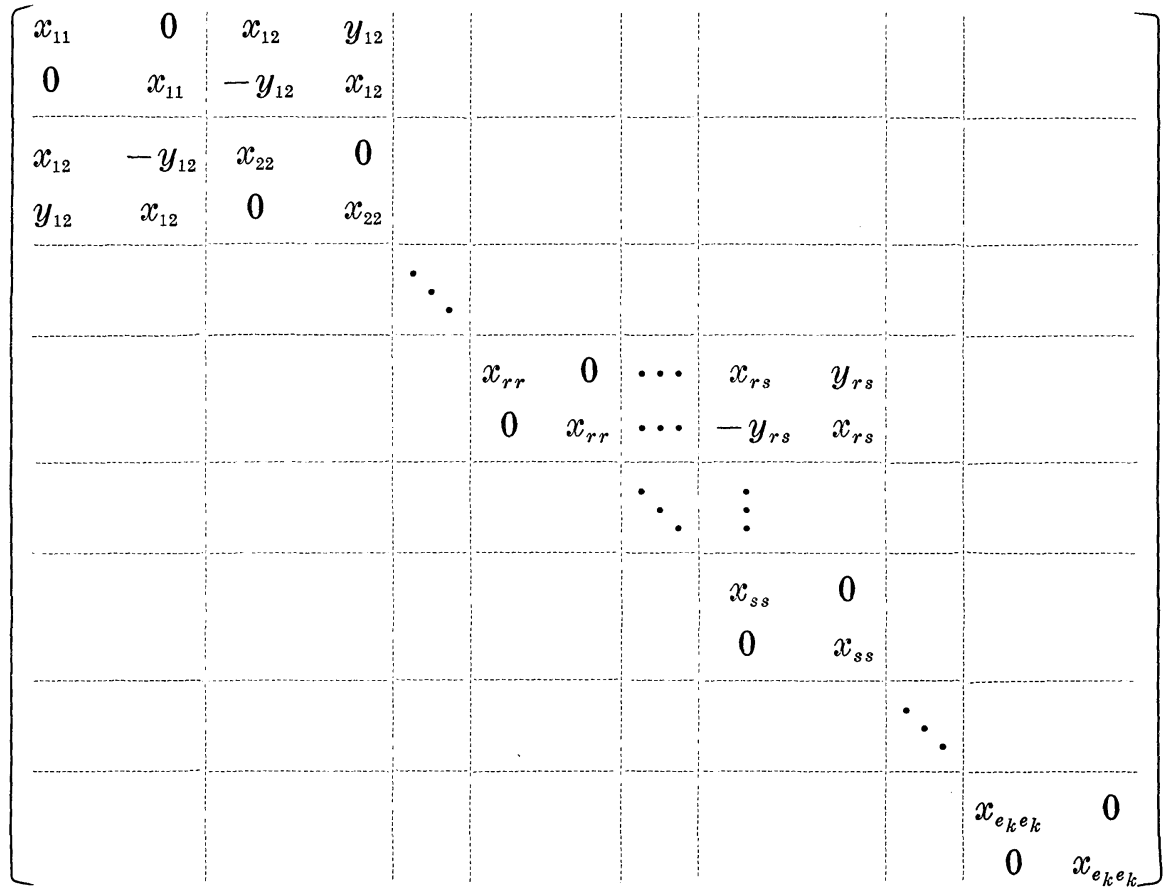

This matrix is of the form $X_{k} \otimes I_{2}+Y_{k} \otimes F$ where $X_{k}=\left(x_{i j}\right)$ is $e_{k^{-}}$ square symmetric and $Y_{k}=\left(y_{i j}\right)$ is $e_{k}$-square skew-symmetric. This completes the proof of Theorem 3 .

3. Some examples. Let $m=2 p$ and let $S_{p}^{\prime}$ be the symmetric group of degree $p$ on $p+1, \cdots, m$. In general if $V$ is a Euclidean space with inner product $(x, y)$ then $V^{m}(H)$ is also a Euclidean space [2] in which the inner product of two symmetric products $x_{1} * \cdots * x_{m}$ and $y_{1} * \cdots * y_{m}$ is given by

$$
\left(x_{1} * \cdots * x_{m}, y_{1} * \cdots * y_{m}\right)=\frac{1}{m !} \sum_{\sigma \in H} \prod_{i=1}^{m}\left(x_{i}, y_{\sigma(i)}\right) .
$$

Set $H=S_{p} \times S_{p}^{\prime}$ (direct product) and define $\varphi \in M_{m}(V, H, R)$ by

$$
\phi\left(x_{1}, \cdots, x_{p}, x_{p+1}, \cdots, x_{m}\right)=\left(x_{1} * \cdots * x_{p}, x_{p+1} * \cdots * x_{m}\right) .
$$

Clearly $\varphi$ is symmetric with respect to $H$ and

$$
\begin{aligned}
\varphi(x, \cdots, x, x, \cdots, x) & =\|x * \cdots * x\|^{2} \\
& \geqq 0 .
\end{aligned}
$$

Moreover $x * \cdots * x=0$ if and only if $x=0$ [2]. Hence $\varphi$ is positive definite. Now suppose that $\phi \in P(H, T)$ where $T: V \rightarrow V$. Then

$$
\varphi\left(T x_{1}, \cdots, T x_{p}, T x_{p+1}, \cdots, T x_{m}\right)=\varphi\left(x_{1}, \cdots, x_{m}\right)
$$


and from (26) we have

(27)

$$
\left(T x_{1} * \cdots * T x_{p}, T x_{p+1} * \cdots * T x_{m}\right)=\left(x_{1} * \cdots * x_{p}, x_{p+1} * \cdots * x_{m}\right) .
$$

It follows from (27) that

$$
K\left(T^{*} T\right)=I
$$

where $T^{*}$ is the adjoint of $T$ and $K(T)$ is the induced transformation in the symmetry class $V^{p}\left(S_{p}\right)$. It is not difficult to show [7] that (28) implies that $T^{*} T=\omega I_{v}$ where $|\omega|=1$. However, since $T^{*} T$ is positive definite, $T^{*} T=I_{V}$, and hence $T$ is orthogonal. It follows that $T$ must have linear elementary divisors over the complex numbers.

In Theorem 1 we proved only that if $P(H, T)$ is nonempty then $T$ has linear elementary divisors corresponding to real eigenvalues. We conjecture that in fact the preceding example is typical in the sense that $T$ always has linear elementary divisors over the complex numbers if $P(H, T)$ is assumed to be nonempty.

We now give an example to show that if $\varphi \in \mathfrak{A}(H, T)$, but $\varphi$ is not positive definite, then the elementary divisors of $T$ over the complex numbers need not be linear. Let $H=S_{2}$ and let $\operatorname{dim} V=4$. Choose $T$ to have

$$
\left(\lambda^{2}+1\right)^{2}
$$

as its only elementary divisor. Then there exists a real basis $E=$ $\left\{e_{1}, \cdots, e_{4}\right\}$ of $V$ so that

$$
[T]_{E}^{E}=\left[\begin{array}{rrrr}
0 & 0 & 0 & -1 \\
1 & 0 & 0 & 0 \\
0 & 1 & 0 & -2 \\
0 & 0 & 1 & 0
\end{array}\right]
$$

Let $A=[T]_{E}^{E}$. Then from (17) it suffices to determine a symmetric matrix $C$ such that

$$
A^{T} C A=C
$$

Define $C$ as follows:

$$
C=\left[\begin{array}{rrrr}
0 & 1 & 0 & -3 \\
1 & 0 & 1 & 0 \\
0 & 1 & 0 & 1 \\
-3 & 0 & 1 & 0
\end{array}\right]
$$

Then $C$ is symmetric (but not positive definite) and (29) is easily 
verified. This example also shows that $P(H, T)$ is empty. It is routine to verify that $\operatorname{dim} \mathfrak{A}(H, T)=1$ in this case but the formula (5) produces the integer 4 .

\section{REFERENCES}

1. A. I. Mal'cev, Foundations of linear algebra, W. H. Freeman, San Francisco, 1963. 2. M. Marcus and H. Minc, Generalized matrix functions, Trans. Amer. Math. Soc. 116 (1965), 316-329.

3. - A Survey of matrix theory and matrix inequalities, Allyn and Bacon, Boston, 1964.

4. S. Perlis, Theory of matrices, Addison-Wesley, 1952.

5. H. J. Ryser, Compound and induced matrices in combinatorial analysis, Proc. of Symposia in Applied Math., Vol. 10, Combinatorial Analysis, Amer. Math. Soc., 1960. 6. I. Schur, Uber endliche Gruppen und Hermitesche Formen, Math. Z. 1 (1918), 184207.

7. Chih-ta, Yen, On matrices whose associated matrices are equal, Acad. Sinica Science Record 1 (1942), 87-90.

Received September 7, 1967, and in revised form May 21, 1969. The work of both authors was supported under grant AFOSR 698-67 of the Air Force Office of Scientific Research.

University of California, SANTA Barbara 


\section{PACIFIC JOURNAL OF MATHEMATICS}

\section{EDITORS}

H. ROYDEN

Stanford University

Stanford, California

Richard Pierce

University of Washington

Seattle, Washington 98105
J. DUGUNDJI

Department of Mathematics

University of Southern California

Los Angeles, California 90007

BASIL GORDON

University of California

Los Angeles, California 90024

\section{ASSOCIATE EDITORS}
E. F. BECKENBACH
B. H. NEUMANN
F. WOLF
K. YosHIDA

\section{SUPPORTING INSTITUTIONS}

UNIVERSITY OF BRITISH COLUMBIA

CALIFORNIA INSTITUTE OF TECHNOLOGY

UNIVERSITY OF CALIFORNIA

MONTANA STATE UNIVERSITY

UNIVERSITY OF NEVADA

NEW MEXICO STATE UNIVERSITY

OREGON STATE UNIVERSITY

UNIVERSITY OF OREGON

OSAKA UNIVERSITY

UNIVERSITY OF SOUTHERN CALIFORNIA
STANFORD UNIVERSITY

UNIVERSITY OF TOKYO

UNIVERSITY OF UTAH

WASHINGTON STATE UNIVERSITY

UNIVERSITY OF WASHINGTON

*

AMERICAN MATHEMATICAL SOCIETY CHEVRON RESEARCH CORPORATION TRW SYSTEMS

NAVAL WEAPONS CENTER 


\section{Pacific Journal of Mathematics}

\section{Vol. 31, No. $1 \quad$ November, 1969}

James Burton Ax, Injective endomorphisms of varieties and schemes........

Richard Hindman Bouldin, A generalization of the Weinstein-Aronszajn

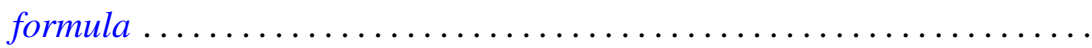

John Martin Chadam, The asymptotic behavior of the Klein-Gordon equation with external potential. II ...............................

Rina Hadass, On the zeros of the solutions of the differential equation

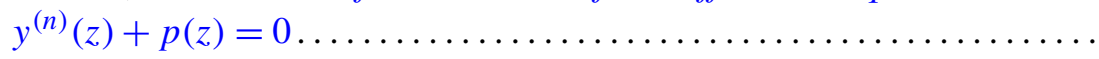

John Sollion Hsia, Integral equivalence of vectors over local modular lattices. II .............................................

Robert Hughes, Boundary behavior of random valued heat polynomial

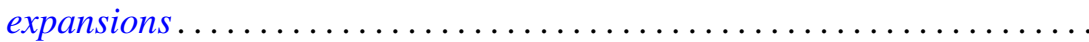

Surender Kumar Jain, Saad H. Mohamed and Surjeet Singh, Rings in which every right ideal is quasi-injective .........................

T. Kawata, On the inversion formula for the characteristic function .........

Erwin Kleinfeld, On right alternative rings without proper right ideals......

Robert Leroy Kruse and David Thomas Price, On the subring structure of

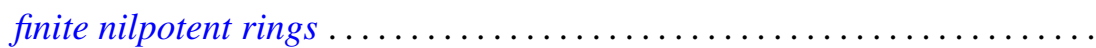

Marvin David Marcus and Stephen J. Pierce, Symmetric positive definite multilinear functionals with a given automorphism. .

William Schumacher Massey, Pontryagin squares in the Thom space of a bundle...

William Schumacher Massey, Proof of a conjecture of Whitney ...

John William Neuberger, Existence of a spectrum for nonlinear transformations

Stephen E. Newman, Measure algebras on idempotent semigroups ...

$\mathrm{K}$. Chandrasekhara Rao, Matrix transformations of some sequence spaces

Robert Bruce Schneider, Some theorems in Fourier analysis on symmetric

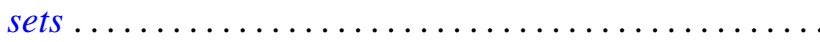

Ulrich F. K. Schoenwaelder, Centralizers of abelian, normal subgroups of hypercyclic groups...

Jerrold Norman Siegel, $G$-spaces, $H$-spaces and $W$-spaces

Robert Irving Soare, Cohesive sets and recursively enumerable Dedekind cuts...

Kwok-Wai Tam, Isometries of certain function spaces .... . .

Awadhesh Kumar Tiwary, Injective hulls of semi-simple modules over regular rings ....

Eldon Jon Vought, Concerning continua not separated by any nonaposyndetic subcontinuum .................... 